\title{
A comprehensive analysis of \#Enuresis conversation on Twitter
}

\author{
Justin $\mathrm{Yu}^{1}$; Adithya Balasubramanian ${ }^{1}$; Jonathan A. Gerber ${ }^{2}$; Abhishek Seth ${ }^{2}$ \\ ${ }^{1}$ Baylor College of Medicine, Houston, TX, United States; ${ }^{2}$ Department of Pediatric Urology, Texas Children's \\ Hospital/Baylor College of Medicine, Houston, TX, United States
}

Cite as: Can Urol Assoc J 2020 February 4; Epub ahead of print. http://dx.doi.org/10.5489/cuaj.6260

Published online February 4, 2020

$* * *$

\begin{abstract}
Introduction: We sought to perform a quantitative and qualitative analysis of online Twitter discussion of enuresis using the hashtag \#Enuresis.

Methods: Symplur, a fee-based Twitter analytics service, was employed to aggregate and analyze Twitter activity, users, and content for \#Enuresis, the official Twitter hashtag for enuresis, between June 2016 and November 2018. Twitter activity was analyzed using average tweets and new users per month. Users were classified based on geographic location, occupation, and organizational affiliation. Content analysis was performed by retrieving information about Twitter engagement metrics, including retweets, links, media, mentions, replies, and frequently used words and hashtags.
\end{abstract}

Results: A total of 3133 tweets and 1555 users utilizing \#Enuresis were identified between June 28, 2016 and November 28, 2018. The average \pm standard deviation [SD] number of tweets using \#Enuresis per month were not significantly different from 2016 through 2018 ( $p=0.292)$. The number of users increased from six to 1555 during the study period, but there was no statistically significant increase in number of new users per month $(\mathrm{p}=0.346)$. Physicians comprised $14 \%$ of the top 100 influencers followed by medical device organizations (13\%). Popular hashtags in \#Enuresis tweets were \#Bedwetting, \#PisEnLaCama, \#schoolnurses, \#helpingkids, \#ninos, and \#salud. Hyperlinks used in \#Enuresis tweets included advocacy, academic, commercial, and other social media websites.

Conclusions: Our analysis of \#Enuresis demonstrates that the online Twitter discussion regarding enuresis is growing. These results indicate that enuresis has a global appeal and has especially gained traction in European countries, as well as in the U.S. 


\section{Introduction}

Enuresis is defined by the International Children's Continence Society (ICCS) as discrete episodes of nocturnal urinary incontinence in children greater than the age of 5 [1] and is a common problem among the pediatric population. It is estimated that $16 \%$ of children at the age of 5 will experience enuresis, which decreases to $13 \%$ at the age of $6,10 \%$ at the age of 7 , and $1 \%$ to $2 \%$ after the age of 15 [2]-[4]. Some studies have found that enuresis can have a detrimental impact on childhood development. For instance, children with enuresis were found to have lower perceptions of self-esteem and self-image [5], [6]. These children may therefore have difficulties adjusting to social situations due to an inability to participate in common activities such as sleepovers, residential school trips, and camping trips [7]. Although there are many management options for enuresis, including bedwetting alarms, motivation therapy, and pharmacologic interventions, the impact of these treatments on self-esteem and patient mental health is an area of active investigation [8].

The Internet has become a widely used resource for patients to obtain medical information, share personal experiences, and garner peer support. Online support groups for conditions such as cancer, mental health disorders, and human immunodeficiency virus (HIV) have been found to be effective in alleviating psychosocial burdens [9]-[11]. While no studies have examined the role of online support groups in pediatric urology, several studies have investigated the role of the internet and social media in this field. Routh et al. in 2009 studied internet content for 10 different pediatric urology conditions, including enuresis, and found that the available online content was high quality for both common and uncommon conditions [12]. Rowe et al. in 2018 demonstrated that social media can be employed as a novel tool for undertaking pediatric urologic focused patient-centered outcomes research [13].

Twitter, a microblogging platform, is a social media service that has emerged as a popular discussion forum for healthcare topics [14]. Conversations on Twitter use hashtags that effectively serve as keywords for topics. Twitter has gained popular appeal amongst both medical professionals and patients. O'Kelly et al. in 2017 identified that parents of pediatric urology patients use social media accounts of medical journals, physicians, and hospitals to access health education information [15]. Many open source efforts including the Urology Tag Ontology Project have aimed to structure the conversation for pediatric urological conditions via hashtags. \#Enuresis was established as the official hashtag for Twitter discussions by the Urology Tag Ontology Project, and has been recognized by urological organizations such as the Urology Care Foundation as well by academic urology journals sponsored by the American Urological Society and European Association of Urology [16]. The goal of this study was to examine the content contained within conversations using \#Enuresis by analyzing users contributing to the conversation, and the content of tweets incorporating the hashtag. 


\section{Methods}

\section{Twitter analysis}

We analyzed the use of \#Enuresis using Symplur, a Twitter Analytics service (www.symplur.com), between June 28, 2016 and November 28, 2018. This time frame included all tweets containing \#Enuresis since the Symplur service began monitoring \#Enuresis. Tweet activity was analyzed by examining number of total users, new users per month, and tweets per month. Tweet metric analysis was performed by obtaining information about retweets as well as tweets with links, embedded media, mentions, and replies.

User information was aggregated via Symplur based on publicly available information. A user profile was generated based on geographic location, occupation, and organizational affiliations. The number of users in North America (Canada, Mexico, United States) were compared against Europe and the rest of the world.

Twitter users employing \#Enuresis were classified into healthcare categories based on profession, organizational affiliation, or credentials using Symplur category definitions [17], [18]. All Symplur classifications were manually verified and corrected if necessary to confirm that stakeholders were accurately identified.

Users were also classified based on influence on the \#Enuresis Twitter discussion. Influence was determined via the SymplurRank metric. SymplurRank is a propriety score that is similar to Impact Factor measurements used by academic journals and controls for Twitter activity that is corrupted by spammers such as number of tweets, retweets and mentions [19]. The Top 100 users with the highest SymplurRank were reported as key influencers of the online discussion.

Tweet content was determined by analyzing words, hashtags, links and the presence of media attachments. Each of these categories were separately investigated to further understand the content within \#Enuresis conversations. The 100 most common words were analyzed along with the Top 25 hashtags and Top 10 Links used in tweets containing \#Enuresis. A survey of tweets containing \#Enuresis as well as any associated hashtags was performed given the fact that tweets may have multiple hashtags.

\section{Statistical analyses}

All statistical tests were undertaken using the R Programming Language 3.5.0 (https://cran.rproject.org/). Two separate analysis of variance (ANOVA) tests were performed to determine differences in \#Enuresis tweet volume and new user adoption in the study time frame. Specifically, the first ANOVA was performed comparing the average number of tweets per month across the 3-year interval, and the second ANOVA was performed comparing the average number of new users per month across the same time interval. The change in number of users was modeled using a linear regression, and the regression coefficient was tested for statistical 
significance. A two-sample, two-sided $t$-test was used to compare the average number of \#Enuresis users in North American countries compared to European countries and to the rest of the world across the 2016 to 2018 time period.

\section{Results}

A total of 3,133 tweets and 1,555 users using the \#Enuresis hashtag were identified since the inception of \#Enuresis monitoring on June 28, 2016. Of the 3,133 tweets, 2,266 were sent with links to other sites $(72 \%), 1,103$ contained media images $(35 \%), 803$ were retweets $(26 \%), 1,150$ were mentions $(37 \%)$, and 18 had replies $(0.57 \%)$. The majority of tweet languages were in English (55\%), followed by Spanish (40\%), German (1.3\%), Indonesian (0.90\%) and Romanian $(0.32 \%)$. There was no statistically significant difference in tweet volume from 2016 to 2018 (p $=0.292$ ) (Figure 1a). The average $\pm \mathrm{SD}$ number of tweets per month containing \#Enuresis was $111 \pm 56$ in 2016, $118 \pm 53$ in 2017, and $85 \pm 45$ in 2018.

The overall number of users using \#Enuresis increased during the study period (Figure 1b). There were initially 6 users in June 2016 that increased in a linear trend to 1,555 users by the end of November $2018\left(\mathrm{R}^{2}=0.96, \mathrm{p}<0.01\right)$. There was no statistically significant difference in the average number $( \pm S D)$ of new users per month $(p=0.346)$. There were $57 \pm 35$ new users in 2016, followed by $60 \pm 37$ in 2017, and $40 \pm 27$ in 2018. Users tweeted from 45 different countries. The majority of known users were located in the Spain (31\%), followed by the United Kingdom (16\%), Mexico (14\%), the U.S. (11\%), and Australia (2\%). North American countries had on average more users, $32.7 \pm 25.3$, compared to the rest of the world, which had $6.5 \pm 19.0$ users on average $(\mathrm{p}=0.029)$. However, when comparing the average number of users in North American countries to European countries $(13.5 \pm 30.7)$, there was no significant difference detected $(\mathrm{p}=0.328)$

The majority of influencers by tweet activity were classified as Doctors (14\%), Organization Medical Devices (13\%), Individual Non-Health (12\%), Organization Advocacy/Support (11\%), and Organization Provider (11\%). These were followed by Organization Media (9\%), Healthcare Providers (5\%), Organization Other Healthcare (5\%), Caregiver/Advocate (4\%), Journalist/Media (3\%), Organization Non-Health (2\%), Individual Other Health (1\%), Organization Research/Academic (1\%), and Researcher/Academic (1\%) (Figure 2).

A word map of the 100 most common words in \#Enuresis tweets is shown in Figure 3. The most common words were "adult" (643 tweets), "enuresis" (377 tweets), "bedwetting" (299 tweets), "children" (290 tweets), "cama" (272 tweets), and "niños" (216 tweets). A detailed overview of associated hashtags used in tweets containing \#Enuresis is shown in Figure 4. The most common hashtags used in conjunction with \#Enuresis were \#Bedwetting (779 tweets), \#PisEnLaCama (158 tweets), \#schoolnurses (93 tweets), \#helpingkids (91 tweet), \#ninos (73 tweets) and \#salud (58 tweets). 
The Top 10 website uniform resource locators (URLs) that were included in tweets with \#Enuresis included thebedwettingdoctor.com (136 shares), pisenlacama.com.ar (114 shares), www.tenscare.co.uk (89 shares), www.dri-sleeper.com (74 shares), www.facebook.com (38 shares), www.eric.org.uk (36 shares), elpais.com (34 shares), www.guiainfantil.com (32 shares), malemmedical.com (30 shares) and onlinelibrary.wiley.com (30 shares).

\section{Discussion}

Social media provides a platform for providers, patients, and healthcare organizations to communicate and share information. Smailhodzic et al. found that social media encouraged equal communication between the patient and physician and increased the rapport of patient-physician relationships [20]. Laranjo et al. found that interventions for patients using social network sites were able to effectively promote health-related behavior change [21]. Farpour et al. described how patients with chronic medical conditions were able to improve their mental health by participating in healthcare interventions that incorporated social media tools [22]. Our study was focused on analyzing Twitter conversations employing \#Enuresis in order to understand existing discussion patterns and highlight avenues to more effectively leverage this platform for improving management of the condition.

There was no significant difference in the average number of monthly tweets containing \#Enuresis across our time period of June 2016 to November 2018 ( $p=0.292)$. This is in contrast to other reported urology Twitter discussions such as \#TesticularCancer [14] and \#KidneyStones [17], which both reported increases over their study periods. One likely explanation is that both testicular cancer and kidney stones impact an older population than enuresis. Testicular cancer in particular is the most common malignancy among young men [23]. As a result, those patients are more likely to have access and be active on the internet and social media compared to pediatric patients afflicted with enuresis.

When analyzing the locations of users tweeting with \#Enuresis, the majority of known users were found to be in Spain and other European countries. There was no difference in the average number of users in North American countries compared to European countries $(\mathrm{p}=0.328)$. This marks a contrast between other hashtag analyses, where the majority of users were located in the United States (US) [14], [17]. The adoption of \#Enuresis across different countries is evidence for the global appeal of Twitter based healthcare conversations. Our results may suggest that users from European and other foreign countries are more willing to engage in enuresis discussion and research compared to users from the US.

There are several reasons why \#Enuresis might have higher engagement levels outside of the US. World Bedwetting Day for example was launched in 2015 by a coalition of international agencies including the ICCS and the European Society for Pediatric Urology [24]. Additionally the ICCS, one of the main research/advocacy groups for the condition, has strong international presence as 9 of the 11 board members reside outside of the US [25]. The fact that these 
advocacy efforts are driven by European members might be an underlying reason for this distribution of users. In addition, the US healthcare system operates largely as a fee-for-service (FFS) model, where payment is distributed based on the quantity of care that is delivered [26]. Since the management of enuresis is non-surgical [27], the economic incentives for enuresis awareness and management may differ from other countries that promote pay-for-performance and integrated care models. Countries with healthcare systems in place that subsidize health maintenance and long-term follow-up may draw more awareness to chronic conditions such as enuresis.

We observed an increase in the number of users from 6 to 1,555 across our study period. Physicians comprised the majority of the top 100 influencers $(14 \%)$, which was followed by medical device organizations (13\%), and advocacy/support organizations (11\%). $79 \%$ of these physicians and 64\% of these advocacy/support organizations tweeted in Spanish, and words such as "niños" and "cama" were in the list of top 10 most commonly used words. These findings are consistent with the international adoption of this hashtag. The relatively high percentage of medical device organizations is likely attributed to the popularity of enuresis prevention technologies. A majority of medical device organizations were related to the manufacture and sale of enuresis alarms, which can be used as a primary treatment for enuresis [28].

A majority of tweets $(72 \%)$ were sent with links. The most commonly tweeted links were affiliated with medical device websites that sold enuresis prevention tools (thebedwettingdoctor.com, www.tenscare.co.uk, https://www.dri-sleeper.com/, malemmedical.com). The next most commonly tweeted websites were advocacy/support sites (pisenlacama.com.ar, www.eric.org.uk, www.guiainfantil.com). Currently there are no studies that have evaluated the effect that online support websites or groups have on alleviating the psychosocial burdens of enuresis. The popularity of these websites in our analysis supports future work to investigate the impact of these internet tools on enuresis management.

We acknowledge that our study has certain limitations. First, we recognize that conversation regarding the condition might exist outside \#Enuresis hashtag. Less than 33\% of \#Enuresis included more colloquial hashtags such as \#Bedwetting or \#PisEnLaCama. This demonstrates that the conversation surrounding \#Enuresis is substantially distinct from the conversation involving these alternative hashtags. Furthermore we wanted to investigate the Twitter conversation surrounding discrete episodes of nocturnal urinary incontinence via hashtags incorporating formal medical terminology in lieu of hashtags using colloquial language such as bed wetting. Second, we recognize that our analysis might be limited by the fact that some Twitter users might not be following traditional Twitter norms and thereby failing to append \#Enuresis to tweets pertaining to this condition. Consequently we acknowledge that our study might therefore under predict the volume of Tweets and number of users discussing this condition on Twitter. Last, due to limitations of the Symplur software, we are unable to correlate 
patient engagement with tweet quality. Emerging evidence has suggested that publications receiving the most media attention may not be the most scientifically rigorous or that the public may place greater value on different subjects than the scientific community[29]. As a result, future research is necessary to determine the quality of information that patients are interacting with.

\section{Conclusions}

Our analysis demonstrates that Twitter is a popular forum for discussions about Enuresis and that many user are employing \#Enuresis to converse about the condition. Our results shows that there have been steady increases in the total number of users who are utilizing this hashtag. Our study indicates that the majority of conversations about \#Enuresis is driven by various influencers including physicians, advocacy groups, and medical device companies. We demonstrate that \#Enuresis has received strong international adoption and that Twitter is a widely used platform for discussing the condition around the globe. 


\section{References}

1. T. Nevéus et al., "The standardization of terminology of lower urinary tract function in children and adolescents: report from the Standardisation Committee of the International Children's Continence Society.," J. Urol., vol. 176, no. 1, pp. 314-24, Jul. 2006.

2. D. M. Fergusson, L. J. Horwood, and F. T. Shannon, "Factors related to the age of attainment of nocturnal bladder control: an 8-year longitudinal study.," Pediatrics, vol. 78, no. 5, pp. 884-90, Nov. 1986.

3. E. Bakker, M. van Sprundel, J. C. van der Auwera, J. D. van Gool, and J. J. Wyndaele, "Voiding habits and wetting in a population of 4,332 Belgian schoolchildren aged between 10 and 14 years.," Scand. J. Urol. Nephrol., vol. 36, no. 5, pp. 354-62, 2002.

4. A. C. Howe and C. E. Walker, "Behavioral management of toilet training, enuresis, and encopresis.," Pediatr. Clin. North Am., vol. 39, no. 3, pp. 413-32, Jun. 1992.

5. P. Phung, G. Kelsberg, and S. Safranek, "Clinical Inquiry: Does primary nocturnal enuresis affect childrens' self-esteem?,” J. Fam. Pract., vol. 64, no. 4, pp. 250-9, Apr. 2015.

6. M. Theunis, E. Van Hoecke, S. Paesbrugge, P. Hoebeke, and J. Vande Walle, "Selfimage and performance in children with nocturnal enuresis.," Eur. Urol., vol. 41, no. 6, p. 660-7; discussion 667, Jun. 2002.

7. E. Fleming, "Supporting children with nocturnal enuresis.," Nurs. Times, vol. 108, no. 41, pp. 22, 24-5.

8. S. A. Redsell and J. Collier, "Bedwetting, behaviour and self-esteem: a review of the literature.," Child. Care. Health Dev., vol. 27, no. 2, pp. 149-62, Mar. 2001.

9. G. Bouma, J. M. Admiraal, E. G. E. de Vries, C. P. Schröder, A. M. E. Walenkamp, and A. K. L. Reyners, "Internet-based support programs to alleviate psychosocial and physical symptoms in cancer patients: a literature analysis.," Crit. Rev. Oncol. Hematol., vol. 95, no. 1, pp. 26-37, Jul. 2015.

10. S. Dosani, C. Harding, and S. Wilson, "Online groups and patient forums.," Curr. Psychiatry Rep., vol. 16, no. 11, p. 507, Nov. 2014.

11. P. K. H. Mo and N. S. Coulson, "Online support group use and psychological health for individuals living with HIV/AIDS.," Patient Educ. Couns., vol. 93, no. 3, pp. 426-32, Dec. 2013.

12. J. C. Routh, E. M. Gong, and C. P. Nelson, "Pediatric urology and the internet--does an uncommon topic decrease content quality?," J. Urol., vol. 182, no. 4, pp. 1569-74, Oct. 2009.

13. C. K. Rowe, M. Shnorhavorian, P. Block, J. Ahn, and P. A. Merguerian, "Using social media for patient-reported outcomes: A study of genital appearance and sexual function in adult bladder exstrophy patients.," J. Pediatr. Urol., vol. 14, no. 4, p. 322.e1-322.e6, Aug. 2018.

14. H. Borgmann et al., "Activity, content, contributors, and influencers of the twitter discussion on urologic oncology.," Urol. Oncol., vol. 34, no. 9, pp. 377-83, 2016.

15. F. O'Kelly et al., "The effect of social media (\#SoMe) on journal impact factor and parental awareness in paediatric urology.," J. Pediatr. Urol., vol. 13, no. 5, p. 513.e1513.e7, Oct. 2017. 
16. A. Kutikov, H. H. Woo, and J. W. Catto, "Urology Tag Ontology Project: Standardizing Social Media Communication Descriptors.," Eur. Urol., vol. 69, no. 2, pp. 183-5, Feb. 2016.

17. J. Salem et al., "Online Discussion on \#KidneyStones: A Longitudinal Assessment of Activity, Users and Content.," PLoS One, vol. 11, no. 8, p. e0160863, 2016.

18. "Healthcare Stakeholders," 2019. [Online]. Available: https://help.symplur.com/general/healthcare-stakeholders.

19. "Symplur Rank," 2019. [Online]. Available: https://help.symplur.com/general/symplurrank.

20. E. Smailhodzic, W. Hooijsma, A. Boonstra, and D. J. Langley, "Social media use in healthcare: A systematic review of effects on patients and on their relationship with healthcare professionals.," BMC Health Serv. Res., vol. 16, p. 442, 2016.

21. L. Laranjo et al., "The influence of social networking sites on health behavior change: a systematic review and meta-analysis.," J. Am. Med. Inform. Assoc., vol. 22, no. 1, pp. 243-56, Jan. 2015.

22. H. R. Farpour, L. Habibi, and S. H. Owji, "Positive Impact of Social Media Use on Depression in Cancer Patients," Asian Pac. J. Cancer Prev., vol. 18, no. 11, pp. 29852988, 2017.

23. M. J. Garner, M. C. Turner, P. Ghadirian, and D. Krewski, "Epidemiology of testicular cancer: an overview.," Int. J. cancer, vol. 116, no. 3, pp. 331-9, Sep. 2005.

24. "World Bedwetting Day | Help \&amp; awareness in treating common condition." [Online]. Available: https://www.worldbedwettingday.com/. [Accessed: 23-Jan-2019].

25. "ICCS - International Children's Continence Society." [Online]. Available: http://i-c-cs.org/. [Accessed: 23-Jan-2019].

26. R. A. Berenson and E. C. Rich, "US approaches to physician payment: the deconstruction of primary care.," J. Gen. Intern. Med., vol. 25, no. 6, pp. 613-8, Jun. 2010.

27. J. Vande Walle et al., "Practical consensus guidelines for the management of enuresis.," Eur. J. Pediatr., vol. 171, no. 6, pp. 971-83, Jun. 2012.

28. T. Neveus et al., "Evaluation of and treatment for monosymptomatic enuresis: a standardization document from the International Children's Continence Society.," J. Urol., vol. 183, no. 2, pp. 441-7, Feb. 2010.

29. E. M. O’Connor, G. J. Nason, F. O’Kelly, R. P. Manecksha, and S. Loeb, "Newsworthiness vs scientific impact: are the most highly cited urology papers the most widely disseminated in the media?," BJU Int., vol. 120, no. 3, pp. 441-454, 2017. 
Figures and Tables

Fig. 1. Analysis of tweet activity (A) and user influx (B) from June 28, 2016 to November 28, 2018 .

Figure 1a. Overview of \#Enuresis Tweet Activity

250
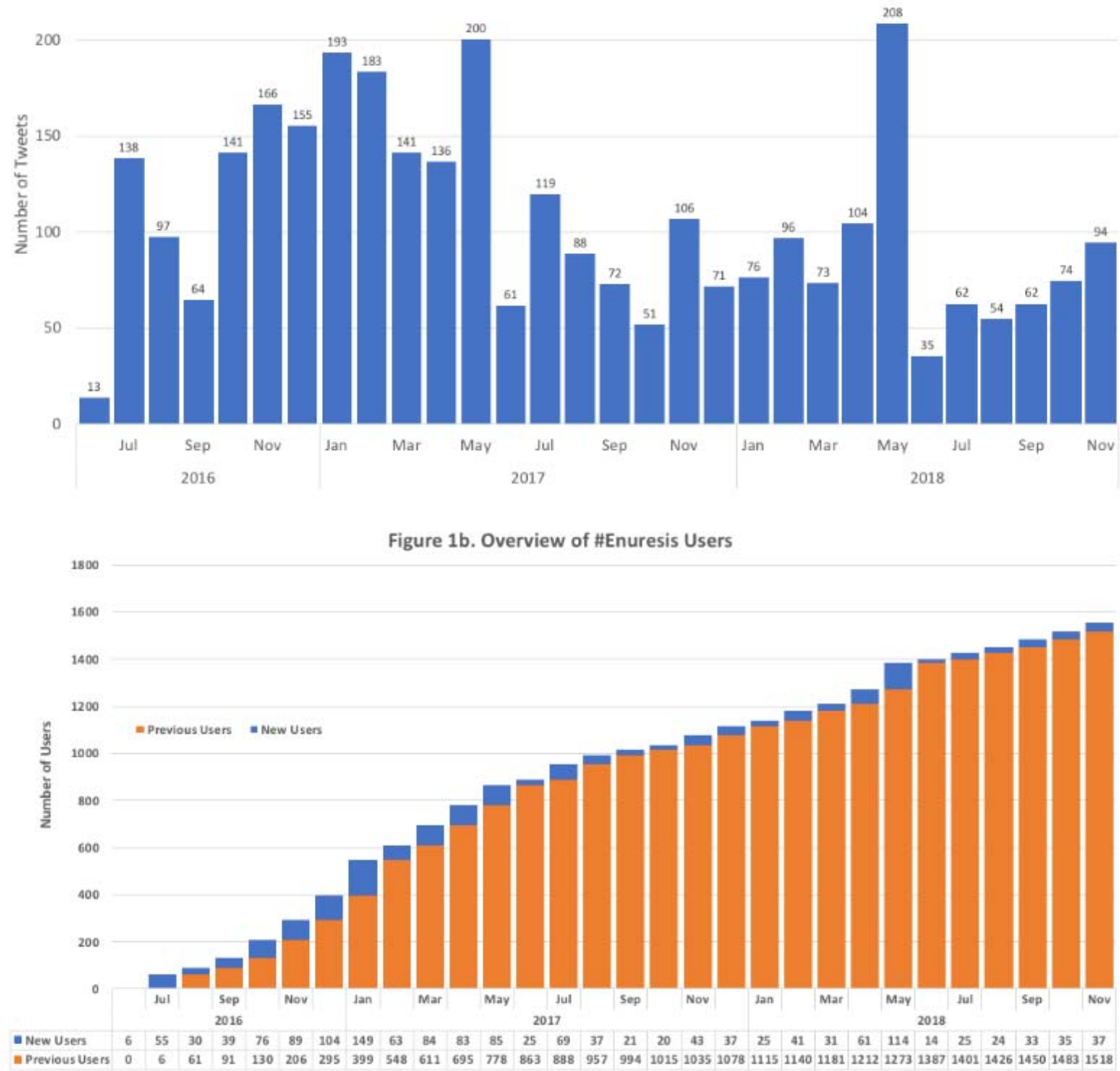
Fig. 2. Distribution of top 100 influencers in \#Enuresis conversation.

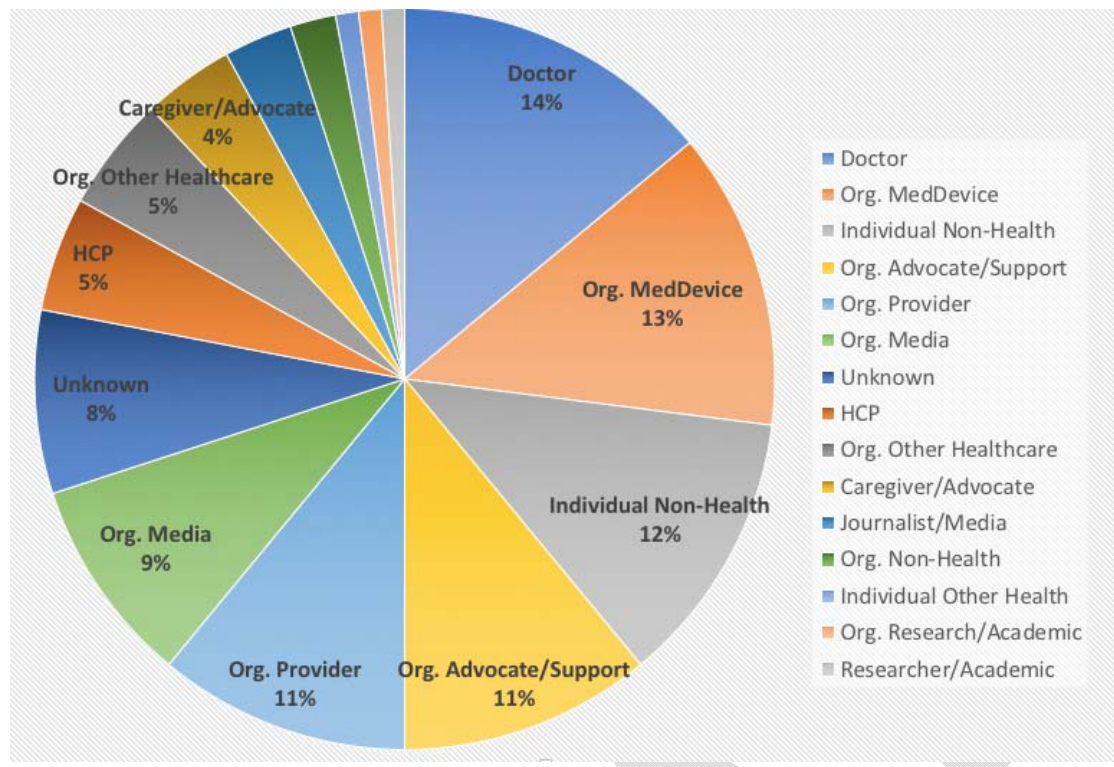

Fig. 3. Cloud map of the top 100 most commonly tweeted words.

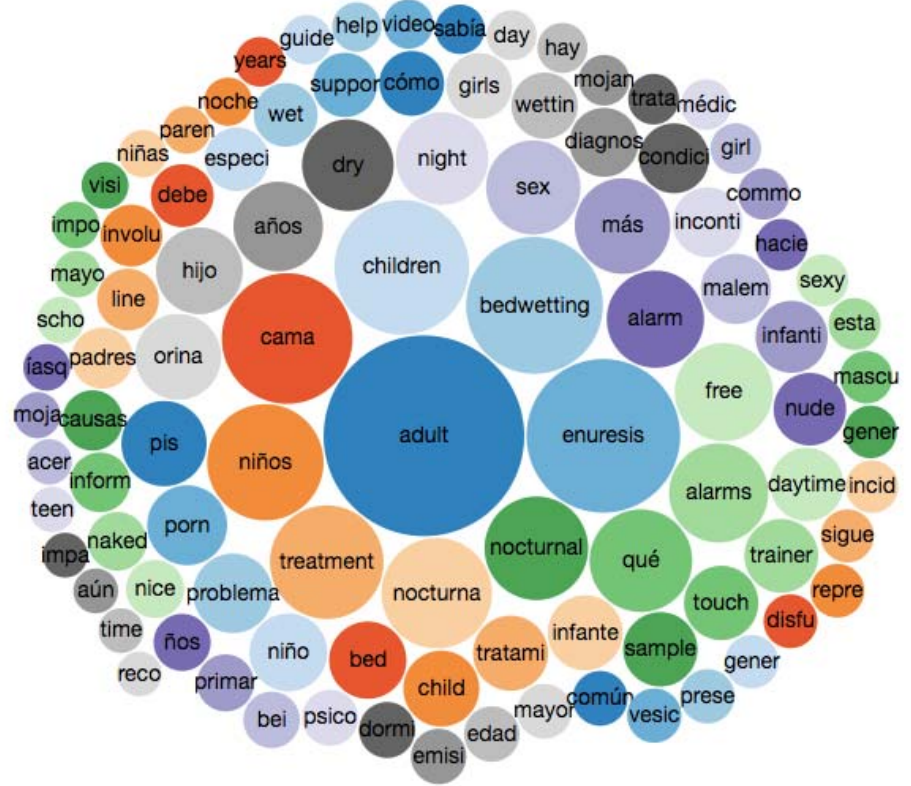

\begin{tabular}{c|c} 
Word & Frequency \\
\hline adult & 643 \\
enuresis & 377 \\
bedwetting & 299 \\
children & 290 \\
cama & 272 \\
ninos & 216 \\
treatment & 206 \\
nocturna & 195 \\
nocturnal & 171 \\
que & 168 \\
alarms & 157 \\
free & 154 \\
alarm & 151 \\
mas & 146 \\
sex & 142 \\
night & 137 \\
dry & 134 \\
anos & 128 \\
hijo & 120 \\
orina & 118 \\
&
\end{tabular}


Fig. 4. Mostly commonly tweeted hashtags in association with \#Enuresis

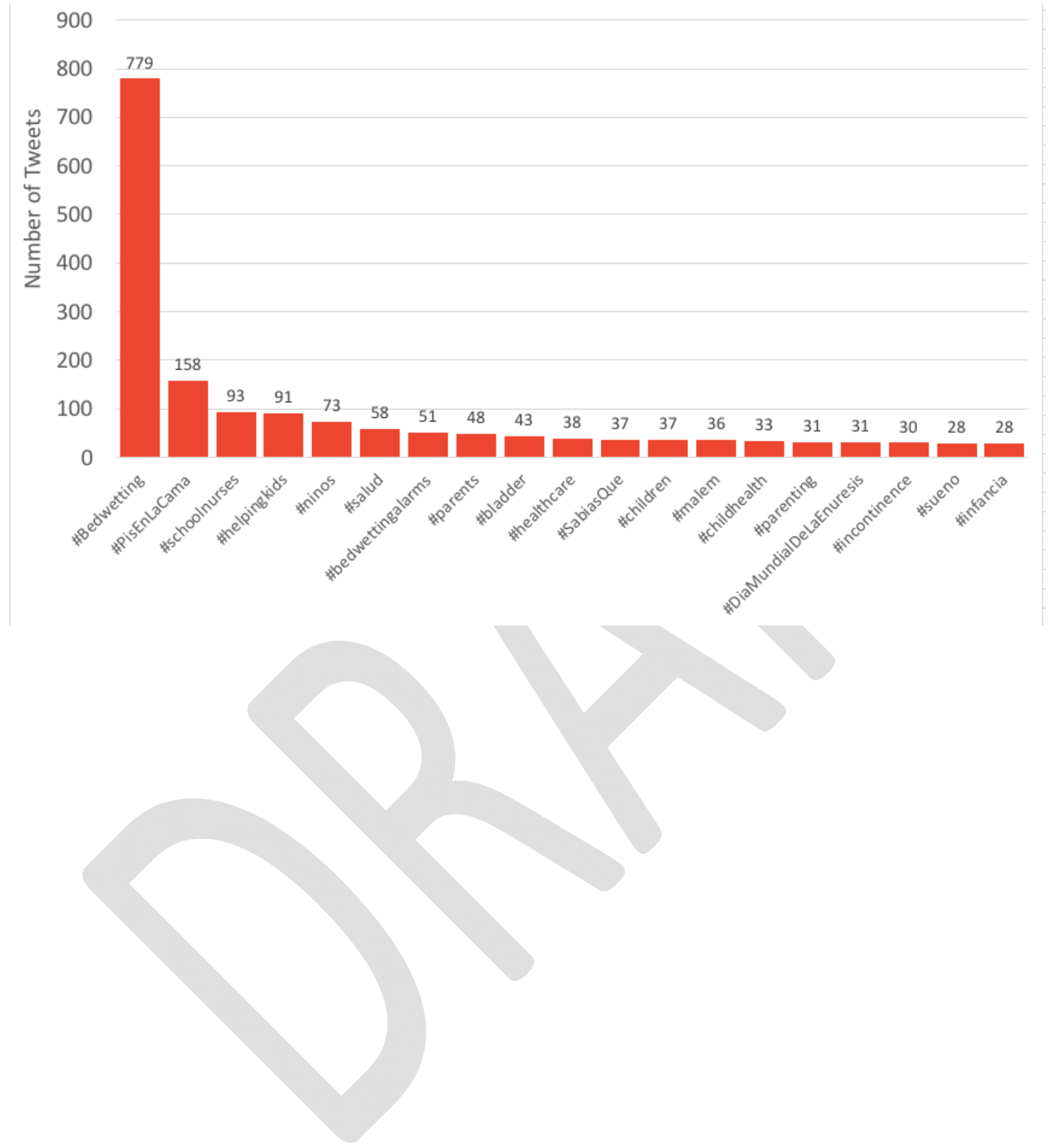

